Please do not remove this page

RMIT

UNIVERSITY

\title{
Demonstration of a numerically optimized resonantly enhanced Mach-zehnder modulator
}

Nguyen, Giang; Visagathilagar, Yuvaraja; Mitchell, Arnan

https://researchrepository.rmit.edu.au/esploro/outputs/9921862015701341/filesAndLinks?institution=61RMIT_INST\&index=null

Nguyen, G., Visagathilagar, Y., \& Mitchell, A. (2006). Demonstration of a numerically optimized resonantly enhanced Mach-zehnder modulator. IEEE Photonics Technology Letters, 18(3), 454-456.

https://doi.org/10.1109/LPT.2005.863174

Published Version: https://doi.org/10.1109/LPT.2005.863174

Repository homepage: https://researchrepository.rmit.edu.au

(c) 2006 IEEE. Personal use of this material is permitted. However, permission to reprint/republish this material for advertising or promotional purposes or for creating new collective works for resale or redistribution to servers or lists, or to reuse any copyrighted component of this work in other works must be obtained from the IEEE.

Downloaded On 2023/04/26 13:19:37 +1000 


\title{
Demonstration of a Numerically Optimized Resonantly Enhanced Mach-Zehnder Modulator
}

\author{
Thach Giang Nguyen, Arnan Mitchell, Member, IEEE, and Yuvaraja S. Visagathilagar, Member, IEEE
}

\begin{abstract}
A practical demonstration of an optimized, resonantly enhanced Mach-Zehnder modulator on $\mathrm{X}$-cut $\mathrm{LiNbO}_{3}$ is presented. Resonant enhancement was achieved by terminating both ends of the electrode with shorted stubs. The modulator electrode active section and inductive terminations have been numerically optimized for resonant operation at $1.8 \mathrm{GHz}$. Suggestions for increasing the frequency of operation are also presented.
\end{abstract}

Index Terms-Electrodes, optical modulation, resonance.

\section{INTRODUCTION}

A Mach-Zehnder modulator (MZM) on $\mathrm{LiNbO}_{3}$ has been widely used in optical communication systems. Conventional modulators which employ a travelling-wave electrode structure offer very large bandwidth but low modulation efficiency. Hence, the radio-frequency (RF) links that use MZMs suffer large link loss. In applications such as radio-on-fiber [1] and local oscillator distribution for photonic mixing [2], a narrow bandwidth around a high frequency carrier is required. Thus, for these applications, it is possible to improve the modulation efficiency of MZM at the expense of modulation bandwidth through resonant electrode structures.

Several demonstrations of resonantly enhanced (RE)-MZMs have been reported [3], [4]. However, these modulators focused on short electrodes to achieve strong resonant enhancement. When reducing the electrode length, the electrooptic interaction length is also reduced, resulting in a comparatively high switching voltage $V_{\pi}$. For many applications, a moderately sized modulator is acceptable. Here resonant enhancement could be used to achieve the best possible efficiency for a specified electrode length. In [5] and [6], resonant enhancement was achieved by placing a single termination at the output port of a travelling wave electrode. This configuration suffers from poor return loss [7] and the resonant enhancement factor is not very strong since the input port still matches to the source. Furthermore, the electrode structure was designed for broad-band operation which is not an optimal structure for RE-MZMs. Oikawa et al. demonstrated a 29.6-mm electrode RE-MZM with double-stub structure [8]. However, $V_{\pi}$ was still quite high due to nonoptimal electrode structure.

To achieve good resonant enhancement, the attenuation of the modulator electrodes needs to be minimized. Furthermore, the inherent $V_{\pi}$ of the electrodes need to be reduced in order to

Manuscript received September 6, 2005; revised November 17, 2005.

The authors are with the School of Electrical and Computer Engineering, Royal Melbourne Institute of Technology, Melbourne, Victoria 3001, Australia (e-mail: s9510300@student.rmit.edu.au; arnan.mitchell@rmit.edu.au; yuvaraja@rmit.edu.au).

Digital Object Identifier 10.1109/LPT.2005.863174

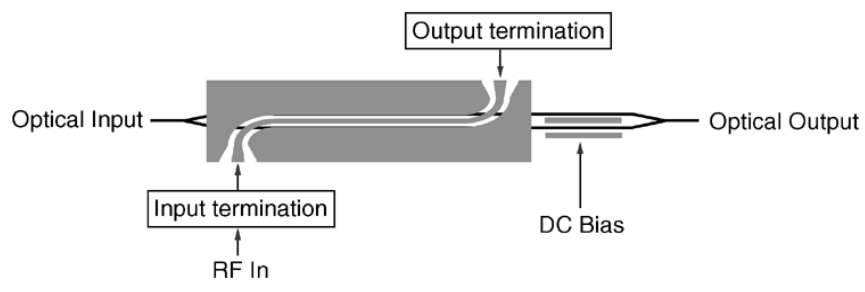

Fig. 1. Resonantly enhanced modulator structure.

maximize modulation efficiency. Therefore, for optimal resonant performance, it is desirable to design the electrode structure that simultaneously provide low $V_{\pi}$ and low loss. In [9], we have theoretically investigated RE-MZM on X-cut $\mathrm{LiNbO}_{3}$ using numerical optimization. It was shown that by optimizing the electrode cross-sectional parameters, low $V_{\pi}$ can be achieved with low electrode loss. In this letter, we demonstrate the experimental realization of the optimal modulator configurations identified in [9].

\section{Device Configuration AND THEORY}

Fig. 1 illustrates the configuration of the resonantly enhanced modulator. By placing the terminations on both input and output ports of the modulator electrode to form a Fabry-Pérot-type cavity [7], most of the microwave signal undergoes multiple reflections inside the cavity to form resonant standing waves. This process results in an enhanced electric field inside the modulator electrode.

The frequency-dependent switching voltage of the modulator is given by

$$
V_{\pi}(f)=\frac{\pi V_{\text {in }}}{|\Delta \Phi|}
$$

where $V_{\text {in }}$ is the voltage amplitude at the modulator input and $\Delta \Phi$ is the total accumulated phase change in the two arms of the modulator. $\Delta \Phi$ is found by the sum of the Pockel's effect

$$
\Delta \Phi=\frac{\pi}{V_{\pi} L} \int_{0}^{L} V(z) e^{j\left(\frac{2 \pi f}{c}\right) n_{o} z} d z
$$

where $V_{\pi}$ is the de switching voltage of the modulator without the terminations, $L$ is the total length of the active section, $V(z)$ is the complex voltage at point $z$ along the active section of the electrode, $n_{0}$ is the effective index of the guided optical mode, and $c$ is the speed of light in the free space.

For broad-band travelling-wave electrode type modulator with impedance match, the voltage inside the active section 
$V(z)$ is smaller than the input voltage $V_{\text {in }}$ due to electrode loss. Hence, the frequency-dependent switching voltage $V_{\pi}(f)$ is always greater than the dc $V_{\pi}$. For the case of RE-MZMs, because of the resonant electrode structure, the voltage inside the active section $V(z)$ can be greater than the input voltage $V_{\text {in }}$. Therefore, $V_{\pi}$ at the resonant frequency can be smaller than the dc $V_{\pi}$.

When an RE-MZM is used in an externally modulated direct detection RF-photonic link assuming small signal operation and quadrature bias, the total system link gain is given by

$$
G(f)=\left(P_{o} \alpha r \frac{\pi}{2 V_{\pi}(f)}\right)^{2}|1+\Gamma|^{2}\left(\frac{50(\Omega) R_{D}}{50(\Omega)+R_{D}}\right)^{2}
$$

where $P_{o}$ is the input optical power, $\alpha$ is the total optical loss, $r$ is the responsivity of the photodetector, $\Gamma=$ $\left(Z_{m}-50(\Omega)\right) /\left(Z_{m}+50(\Omega)\right)$ is the voltage reflection coefficient at the modulator input, $Z_{m}$ is the modulator impedance at frequency $f$, and $R_{D}$ is the termination resistance of the photodetector. The link is assumed to be terminated at $50-\Omega$ input and output.

\section{RESONANT MODULATOR REALIZATION}

In [9], the optimal electrode configuration to maximize RE-MZM efficiency has been identified. The gap between the hot electrode and ground plane is $50 \mu \mathrm{m}$. The width and the thickness of the electrode are 22 and $30 \mu \mathrm{m}$, respectively. The $\mathrm{SiO}_{2}$ buffer layer thickness is $0.2 \mu \mathrm{m}$. The electrode has a 3.7-cm-long active section.

The modulator was fabricated in $\mathrm{X}$-cut $\mathrm{LiNbO}_{3}$. The optical waveguide was formed by diffusion of $850-\AA$-thick and $7-\mu \mathrm{m}$ -wide Titanium strip at $1010^{\circ} \mathrm{C}$ for $10 \mathrm{~h}$. The fabricated modulator was then characterized using a microprobe station setup with a vector network analyzer. The $S_{11}$ and $S_{21}$ parameters of the modulator electrode were measured. At the same time, basic optical and dc modulation characteristics were also measured. From these measured parameters, the characteristics of the fabricated modulator without terminations were calculated. The electrode has attenuation constant $\alpha=0.21 \mathrm{~dB} / \mathrm{cm} \sqrt{\mathrm{GHz}}$, characteristic impedance $Z_{0}=36 \Omega$, effective index $N_{m}=$ 3.23 , and dc switching voltage $V_{\pi}=2.5 \mathrm{~V}$.

To resonantly enhance the fabricated modulator, inductive terminations were added at either end to form a resonant cavity. The inductive terminations used in this investigation were realized by shorted coplanar waveguide stub circuits on ceramic substrate, as shown in Fig. 2. The gap-width-gap ratio for the input-output section and the shorted stub are 180-380-180 $\mu \mathrm{m}$ and $500-10-500 \mu \mathrm{m}$, respectively. The terminations were formed by gold electroplating with gold thickness of $10 \mu \mathrm{m}$ on ceramic substrate. This configuration results in $50-\Omega$ impedance in the input and output sections and 145- $\Omega$ impedance in the stub sections. Using the same optimization procedure as in [9], the input and output stub lengths were optimized to provide maximum cavity resonance at the operational frequency of $1.8 \mathrm{GHz}$. The fabricated terminations were then attached to the modulator electrode tapers by wire-bonding.

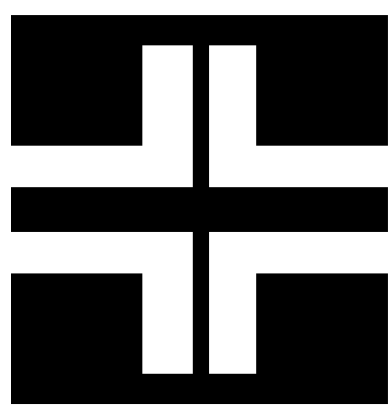

(a)

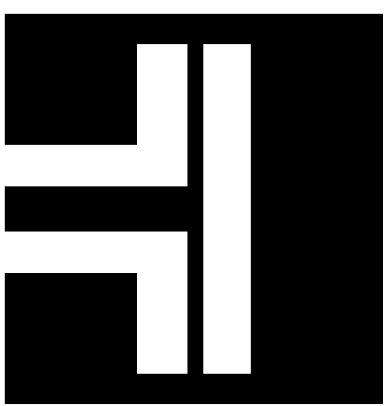

(b)
Fig. 2. (a) Input termination and (b) output termination.

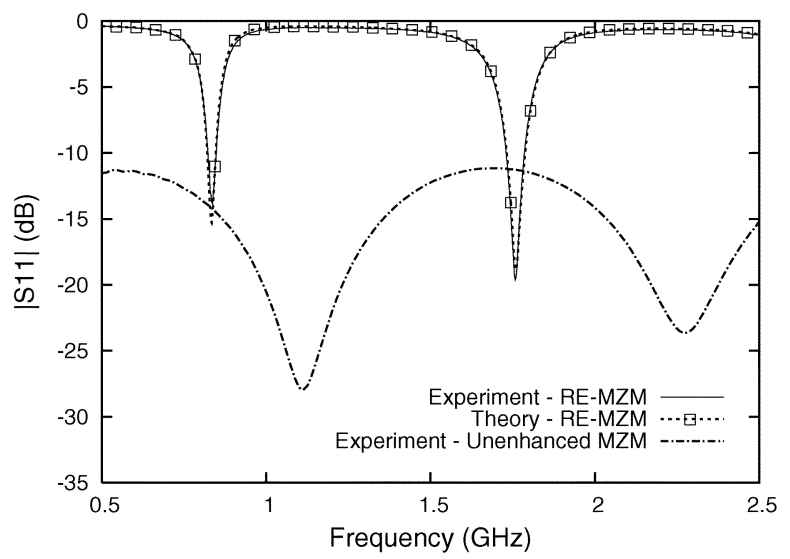

Fig. 3. Link return loss of the RF-photonic links using different modulators.

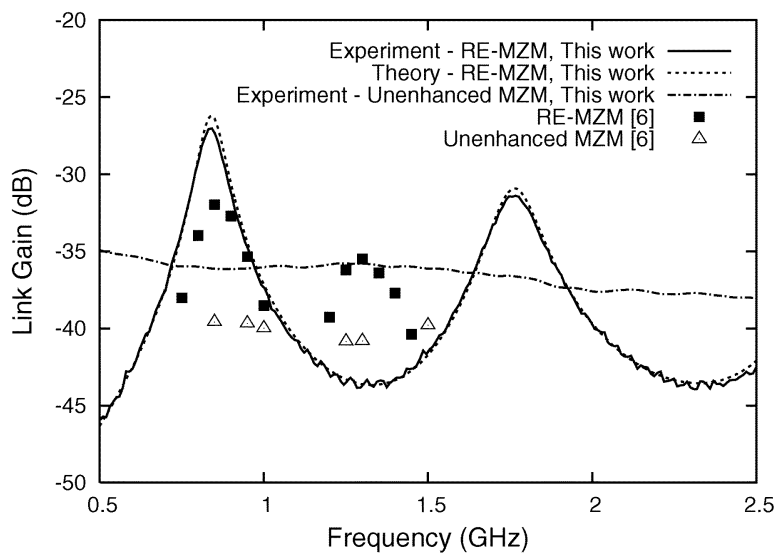

Fig. 4. Link gain of the RF-photonic links using different modulators.

\section{EXPERIMENTAL RESULTS}

Fig. 3 presents the measured return loss $\left|S_{11}\right|$ of the RE-MZM and that of the same basic modulator with the resonant terminations removed and being terminated with $50-\Omega$ load. It can be seen that at the resonant frequency, the RE-MZM exhibits a much lower return loss than the unenhanced modulator. This indicates that a good resonance was achieved. The return loss calculated from the simulation is also presented in Fig. 3 for comparison with the measured data. Good agreement was achieved between theoretical prediction and measurement.

In Fig. 4, the link gain of the RF-photonic links formed using the RE-MZM, the unenhanced basic modulator (with terminations removed) as a function of frequency is shown. At the designed frequency of $1.8 \mathrm{GHz}$, with the inductive terminations 


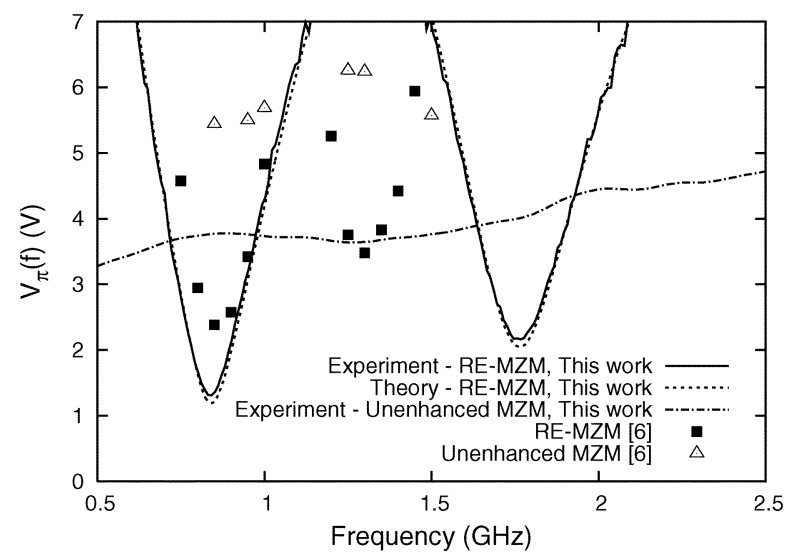

Fig. 5. Frequency-dependent switching voltages $V_{\pi}(f)$ of different modulators.

added, the RE-MZM exhibits over 5-dB improvement over the unenhanced modulator (with the terminations removed) with a 3 -dB bandwidth of about $11 \%$. It can also be seen that apart from the designed resonant frequency of $1.8 \mathrm{GHz}$, there is first-order resonance at around $0.85 \mathrm{GHz}$. At this frequency, the RE-MZM shows about 9-dB improvement over the unenhanced modulator. However, the return loss is not as good as at $1.8 \mathrm{GHz}$ since the terminations are not optimized to operate at that frequency.

Also shown in Fig. 4 are the results calculated from the data reported in [6], where the unenhanced modulator was a z-cut broad-band travelling-wave modulator and the resonant enhancement was achieved by open circuit termination with a length of coaxial cable to tune the resonant frequency. The resonantly enhanced modulator in [6] shows two resonant frequencies at 0.85 and $1.3 \mathrm{GHz}$ with enhancement factors of 7.6 and $5.3 \mathrm{~dB}$, respectively.

In comparison with the RE-MZM demonstrated in [5] and [6], the RE-MZM in this letter shows better resonant enhancement since the modulator electrode is terminated at both ends to form a true resonant cavity and the terminations were optimized to maximize the resonant enhancement. Furthermore, the electrode structure was optimized for resonant operation; hence, the overall modulation efficiency at the resonant frequency is much better than that of a resonant modulator that utilizes a standard broad-band travelling electrode.

In Fig. 5, the frequency-dependent $V_{\pi}(f)$ of the resonantly enhanced, unenhanced modulators and the results reported in [6] are plotted. The experimental $V_{\pi}(f)$ were derived from the measured link gain using (3). The theoretical $V_{\pi}(f)$ for the RE-MZM was calculated using (1). There is good agreement between the experimental and theoretical values. It can be seen that at the resonant frequency, $V_{\pi}$ of the RE-MZM is much lower than that of the unenhanced modulator. Furthermore, at resonance, $V_{\pi}$ is lower than the dc $V_{\pi}$ of the unenhanced modulator.

\section{DISCUSSION}

The photonic link gain demonstrated in Fig. 4 represents a significant improvement of a travelling-wave modulator at $1.8 \mathrm{GHz}$. It would be of interest to explore the possibility of achieving similar resonant enhancement at higher frequencies for application in broad-band fiber-wireless, advanced modulation format communications and also photonic mixing and local oscillator distribution. The enhancement that can be achieved at higher frequencies will be limited by electrode attenuation, a new numerical optimization as presented in [9] would be required. At higher frequencies it will be critical that the inductive terminations are as compact as possible and are integrated close to the active region of the modulator. To achieve this, high- $Q$ inductive terminations should be realized, for example using spiral inductors. These could then be flip-chip interfaced to the modulator active region [10]. The same optimization approach can also be applied to the double-stub structure [4], [8] to optimize the electrode configuration. It is anticipated that with optimized electrode structure, further improvement to the resonant enhancement can be achieved.

\section{CONCLUSION}

In this letter, we have experimentally demonstrated a resonantly enhanced modulator using an optimal electrode configuration that has been theoretically investigated previously. The demonstrated modulator has low electrode loss and low $V_{\pi}$. With the use of shorted-stub terminations to form Fabry-Pérottype microwave cavity, the realized modulator exhibits 9-dB improvement over an equivalent broad-band device and 5-dB improvement over the same basic modulator, but without resonant terminations. The design and realization of equally efficient resonant modulators at higher frequencies is currently under investigation.

\section{REFERENCES}

[1] A. Nirmalathas, D. Novak, C. Lim, R. Waterhouse, and D. Castleford, "Fiber networks for wireless applications," in Conf. Proc. LEOS 2000 Annual Meeting, vol. 1, 2000, pp. 35-36.

[2] A. Lindsay, G. Knight, and S. Winnall, "Photonic mixers for wide bandwidth RF receiver applications," IEEE Trans. Microw. Theory Tech., vol. 43, no. 9, pp. 2311-2317, Sep. 1995.

[3] R. Krahenbuhl and M. Howerton, "Investigations on short-path-length high-speed optical modulators in $\mathrm{LiNbO}_{3}$ with resonant-type electrodes," J. Lightw. Technol., vol. 19, no. 9, pp. 1287-1297, Sep. 2001.

[4] T. Kawanishi, S. Oikawa, K. Higuma, Y. Matsuo, and M. Izutsu, "Resonant electrode structures for band-operation-type optical modulators," in Proc. SPIE, vol. 4870, 2002, pp. 577-586.

[5] G. Gopalakrishnan and W. Burns, "Performance and modeling of resonantly enhanced $\mathrm{LiNbO}_{3}$ modulators for low-loss analog fiber-optic links," IEEE Trans. Microw. Theory Tech., pt. 1-2, vol. 42, no. 12, pp. 2650-2656, Dec. 1994.

[6] M. Howerton, W. Burns, and G. Gopalakrishnan, "SBS suppression using a depolarized source for high power fiber applications," J. Lightw. Technol., vol. 14, no. 3, pp. 417-422, Mar. 1996.

[7] Y. Visagathilagar, A. Mitchell, and R. Waterhouse, "Fabry-perot type resonantly enhanced Mach-Zehnder modulator," in Int. Topical Meeting Microwave Photonics (MWP'99), vol. 1, 1999, pp. 17-20.

[8] S. Oikawa, T. Kawanishi, K. Higuma, Y. Matsuo, and M. Izutsu, "Double-stub structure for resonant-type optical modulators using 20- $\mu$ m-thick electrode," IEEE Photon. Technol. Lett., vol. 15, no. 2, pp. 221-223, Feb. 2003.

[9] T. G. Nguyen, A. Mitchell, and Y. S. Visagathilagar, "Investigation of resonantly enhanced modulators on $\mathrm{LiNbO}_{3}$ using FEM and numerical optimization technique," J. Lightw. Technol., vol. 22, no. 2, pp. 526-533, Feb. 2004.

[10] Y. Visagathilagar, W. Rowe, A. Mitchell, G. Bennett, and M. Grosser, "Packaging of a high-speed optical modulator using flip chip interconnects," in Proc. Electronic Components and Technology (ECTC 2004), vol. 1, 2004, pp. 212-218. 